\title{
OSCILLATIONS OF THE STATIC MESON FIELDS AT FINITE BARYON DENSITY
}

\author{
Wojciech Florkowski $^{a}$ and Bengt Friman ${ }^{b, c}$ \\ a H. Niewodniczański Institute of Nuclear Physics \\ ul. Radzikowskiego 152, 31-342 Kraków, Poland \\ $b \quad$ Gesellschaft für Schwerionenforschung GSI \\ Postfach 110552, D-64220 Darmstadt, Germany \\ c Institut für Kernphysik, Technische Hochschule Darmstadt \\ D-64289 Darmstadt, Germany
}

\begin{abstract}
The spatial dependence of static meson correlation functions at finite baryon density is studied in the Nambu - Jona-Lasinio model. In contrast to the finite temperature case, we find that the correlation functions at finite density are not screened but exhibit long-range oscillations. The observed phenomenon is analogous to the Friedel oscillations in a degenerate electron gas.
\end{abstract}




\section{Introduction}

Recently, there is a growing interest in studying the properties of hadronic correlation functions. The correlation functions form a common ground, where QCD inspired effective theories can be tested against each other as well as against lattice results, where available [1].

The hadronic correlation functions have been intensively studied in lattice simulations of QCD at finite temperatures [2, 3, 4, 5]. The results of these simulations show that at large distances the correlation functions decrease exponentially. The screening masses characterizing this type of behaviour are, at $T>T_{c}$, degenerate for chiral partners. This reflects the restoration of chiral symmetry at high temperatures. Moreover, as it was pointed out by Eletskii and Ioffe [6] (see also [7]), the values of the screening masses are close to $2 \pi T$ for mesons and $3 \pi T$ for baryons. Such results are typical for a gas of non-interacting quarks. Therefore, the lattice results show that at high temperatures quarks behave, to large extent, as free particles.

The results of the lattice simulations have been interpreted in terms of simple models by several authors: Gocksch [8] argues that the hadronic screening masses can be well described in the linear sigma model; Hansson and Zahed [9] claim that the static correlation functions in high-temperature QCD can be well calculated from an equivalent problem of non-relativistic quarks in a dimensionally reduced theory; similarly, V. Koch et al. [10 argue that the propagation of a light fermion in a spatial direction at high temperatures can be described effectively by a two-dimensional Schrödinger equation.

In Ref. [11] the temperature dependence of the meson screening masses was computed and discussed in the framework of the Nambu - Jona-Lasinio (NJL) model. The results are in qualitative agreement with the lattice simulations. In the present paper, we explore the static meson correlation functions at finite baryon density, using the methods developed in [7, 11]. In this way, we complement and generalize our previous work. We note that, for studying systems at finite baryon density one has to rely on models, since lattice results are available only for vanishing density.

The NJL model was introduced already in the early sixties as a theory of interacting nucleons [12]. Later it was reformulated in terms of quark degrees of freedom. Numerous calculations demonstrate the success of the model in describing hadronic data (for a recent review see one of the articles in [13). Two general characteristics of QCD are described by the model, namely the chiral invariance which is spontaneously broken in the ground state and the chiral phase transition (the chiral symmetry is restored at high temperature or density). On the other hand, the model is not renormalizable and not confining. Thus, it is an effective theory, valid only at low energies for phenomena, which are controlled by the chiral symmetry of QCD.

Since the NJL model exhibits a chiral phase transition, it is very instructive to use it in order to study the temperature (density) dependence of various physical quantities like, e.g., the quark condensate [14], the pion decay constant [15, 16], and the dynamic masses of hadrons [14, 16, 17]. The model has also been used to investigate the thermodynamic properties of the quark-meson plasma near the chiral phase transition [18].

In this paper we study the in-medium static meson correlation functions at finite baryon density. In contrast to the behaviour known from the high temperature calculations, we find 
that the correlation functions at finite baryon density are not screened but oscillate in space. This behaviour is analogous to the Friedel oscillations in a degenerate electron gas [20]; they are caused by the existence of the sharp Fermi surface.

We find that the NJL approach to the correlation functions breaks down already at moderate densities. Thus, to complete the picture we compute the meson correlation function at higher densities in perturbative QCD. Keeping only the leading term, we find oscillations with a period $\delta r=\pi / p_{F}$, where $p_{F}$ is the Fermi momentum of the quark sea.

The paper is organized as follows. In the next section we define the model. In Section 3 the details of the calculations are presented. Section 4 contains our results on the density dependence of the quark and meson masses. In Section 5 we discuss our results on the correlation function. The results based on the perturbative QCD are presented in Section 6 . We summarize the paper in Section 7.

\section{Definition of the model}

Our calculations are based on the following form of the Lagrangian

$$
\mathcal{L}=\bar{\psi}\left(i \gamma_{\mu} \partial^{\mu}-m\right) \psi+\sum_{a=0}^{3} \frac{G_{S}}{2}\left[\left(\bar{\psi} \sigma_{a} \psi\right)^{2}+\left(\bar{\psi} i \gamma_{5} \sigma_{a} \psi\right)^{2}\right] .
$$

Here $\psi$ is the Dirac field with additional flavour $\left(N_{f}=2\right)$ and colour $\left(N_{c}=3\right)$ degrees of freedom, $\sigma_{a}$ are the Pauli matrices (with $\sigma_{0}=1$ ), $G_{S}$ is the coupling constant, and $m$ is the current quark mass $\left(m_{u}=m_{d}=m\right)$.

In the chiral limit, $m \rightarrow 0$, the Lagrangian (1) is invariant under the unitary transformations $\mathrm{U}_{V}(1), \mathrm{U}_{A}(1)$ and the chiral symmetry $\mathrm{SU}_{L}(2) \times \mathrm{SU}_{R}(2)$. These are fundamental symmetries of QCD, the underlying theory of strong interactions. In the QCD vacuum the axial $\mathrm{U}_{A}(1)$ symmetry is broken due to the instanton effects [19 and $\mathrm{SU}_{L}(2) \times \mathrm{SU}_{R}(2)$ is spontaneously broken down to $\mathrm{SU}_{V}(2)$. In the Nambu-Jona-Lasinio model the spontaneous breaking of the chiral symmetry is reproduced. The explicit breaking of the $\mathrm{U}_{A}(1)$ symmetry, accounting for the instanton effects, can be modelled by adding an extra term to the Lagrangian (1). In order to keep things as simple as possible, we do not take this term into account here. This does not affect our results, since we do not consider the $\eta-\eta^{\prime}$ channel.

The self-energy of quarks is obtained in the Hartree-Fock approximation by a selfconsistent solution of the Schwinger-Dyson equation

$$
\begin{aligned}
\Sigma= & G_{S} i \sum_{a=0}^{3} \int \frac{d^{4} p}{(2 \pi)^{4}}\left[\sigma_{a} \operatorname{Tr}\left[\sigma_{a} S(p)\right]-\sigma_{a} S(p) \sigma_{a}\right. \\
& \left.+i \sigma_{a} \gamma_{5} \operatorname{Tr}\left[i \sigma_{a} \gamma_{5} S(p)\right]-i \sigma_{a} \gamma_{5} S(p) i \sigma_{a} \gamma_{5}\right] \\
= & G_{S} i \sum_{a=0}^{3} \int \frac{d^{4} p}{(2 \pi)^{4}} \sigma_{a} \operatorname{Tr}\left[\sigma_{a} S(p)\right] .
\end{aligned}
$$

Here Tr denotes the trace over flavour, colour and spinor indices, and $S(p)$ is the quark 
propagator

$$
S(p)^{-1}=\not p-\Sigma-m+i \epsilon
$$

Using the last expression we define the quark condensate in the following way

$$
\langle\bar{q} q\rangle=-\frac{i}{2} \operatorname{Tr} S\left(x=0^{-}\right)=-4 N_{c} i \int \frac{d^{4} p}{(2 \pi)^{4}} \frac{\Sigma+m}{p^{2}-(\Sigma+m)^{2}+i \epsilon} .
$$

The sum $M=\Sigma+m$ is the constituent quark mass. Eqs. (2) and (国) lead to a simple relation between $M$ and the condensate

$$
M=m-2 G_{S}\langle\bar{q} q\rangle .
$$

The zeroth-order correlation function is defined by the expression

$$
\chi_{A A}^{(0)}(Q)=2 i N_{c} \operatorname{Sp} \int \frac{d^{4} p}{(2 \pi)^{4}}\left[\Gamma_{A} S(p+Q) \Gamma_{A} S(p)\right] .
$$

Here $A=P(A=S)$ corresponds to the pseudoscalar (scalar) channel, $Q^{\mu}=(\omega, \mathbf{q})$ is the external momentum, $\Gamma_{P}=i \gamma_{5}, \Gamma_{S}=1$, and Sp denotes the trace over the spinor indices. In the random phase approximation the full correlation function has the form

$$
\chi_{A A}(Q)=\frac{\chi_{A A}^{(0)}(Q)}{1-G_{S} \chi_{A A}^{(0)}(Q)} .
$$

It is important to realize that the in-medium correlation function $\chi_{A A}(Q)$ depends on the variables $\omega^{2}$ and $\mathbf{q}^{2} \equiv q^{2}$ separately. From now on we shall use the notation $\chi_{A A}\left(\omega^{2}, q^{2}\right)$ in order to exhibit this dependence explicitly. The dynamic mass is defined by the position of the lowest lying pole of $\chi_{A A}\left(\omega^{2}, 0\right)$. In our case it is easily found by solving the equation

$$
1-G_{S} \chi_{A A}^{(0)}\left(m_{d y n}^{2}, 0\right)=0 .
$$

The screening mass, on the other hand, is defined by the asymptotic behavior of the static correlation function in r-space, namely

$$
m_{s c r}=-\lim _{r \rightarrow \infty} \frac{d \ln \chi_{A A}(r)}{d r}
$$

where

$$
\chi_{A A}(r)=\int \frac{d^{3} q}{(2 \pi)^{3}} \chi_{A A}\left(0, q^{2}\right) e^{i \mathbf{q} \cdot \mathbf{r}}=\frac{1}{4 \pi^{2} i r} \int_{-\infty}^{+\infty} d q q \chi_{A A}\left(0, q^{2}\right) e^{i q r}
$$

In Eq. (10) the angular integrals were performed using the fact that $\chi_{A A}\left(0, q^{2}\right)$ is a function of $q^{2}$ only. In vacuum, the dynamic and screening masses are equal due to Lorentz invariance. 
On the other hand, at finite temperature or density the two masses can be different, since the heat bath introduces a preferable reference frame. The relation between the dynamic and the screening masses is discussed more thoroughly in [11]. The main purpose of this paper is to compute the static correlation functions defined by (10).

\section{Physical quantities at finite density}

\section{i) Imaginary time formalism}

In our calculations we adopt the imaginary time formalism [20, 21]. Formally, this is achieved by the following substitution [21]

$$
\int \frac{d^{4} p}{(2 \pi)^{4}} f\left(p^{0}, \mathbf{p}\right) \longrightarrow \int_{-i \infty}^{+i \infty} \frac{d p^{0}}{2 \pi} \int \frac{d^{3} p}{(2 \pi)^{3}} f\left(p^{0}, \mathbf{p}\right)+\oint_{\mathcal{C}} \frac{d p^{0}}{2 \pi} \int \frac{d^{3} p}{(2 \pi)^{3}} f\left(p^{0}, \mathbf{p}\right) .
$$

Here $\mathcal{C}$ denotes an integration contour in the complex energy plane $p^{0}$. The position of the contour is fixed by the value of the chemical potential $\mu$, see Fig. 1 . In the case when the integrand depends additionally on the external frequency, e.g., $f=f\left(p^{0}, \mathbf{p}, \omega\right)$, the integrals on the right hand side of formula (11) should be evaluated for purely imaginary values of $\omega$ and subsequently analytically continued to real frequencies.

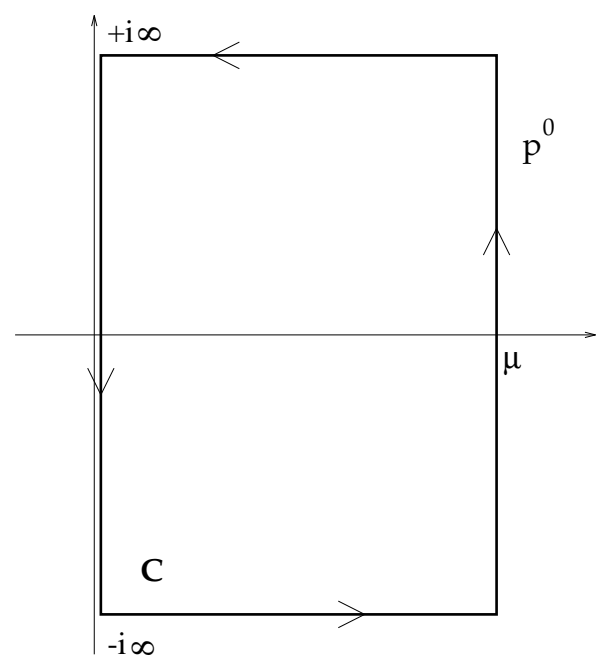

Figure 1: Integration contour in the complex energy plane used for the evaluation of the matter parts .

The advantage of the decomposition (11) is that we can separate the so called vacuum part from the matter part. The vacuum part of a physical quantity does not explicitly depend on the occupation of phase space and reduces at $T=\mu=0$ to its vacuum expectation value. 
On the other hand, the matter part depends explicitly on the occupation of phase space and consequently vanishes in vacuum.

Using Eqs. (4) and (11) we can decompose the condensate into two parts

$$
\langle\bar{q} q\rangle=\langle\bar{q} q\rangle_{v a c}+\langle\bar{q} q\rangle_{m a t} .
$$

In the similar way, using Eqs. (6) and (11) we find

$$
\chi_{A A}^{(0)}\left(\omega^{2}, q^{2}\right)=\chi_{A A, v a c}^{(0)}\left(Q^{2}\right)+\chi_{A A, m a t}^{(0)}\left(\omega^{2}, q^{2}\right) .
$$

ii) Vacuum parts

In this subsection we recapitulate our calculation of the vacuum parts (more details can be found in [1]). After a Wick rotation of the variables $p^{0}=i p_{4}$ and $\omega=i q_{4}$, we find

$$
\begin{gathered}
\langle\bar{q} q\rangle_{v a c}=-\frac{M}{2} I_{1, v a c}\left(M^{2}\right), \\
\chi_{P P, v a c}^{(0)}\left(-q_{E}^{2}\right)=I_{1, v a c}\left(M^{2}\right)+q_{E}^{2} I_{2, v a c}\left(M^{2}, q_{E}^{2}\right)
\end{gathered}
$$

and

$$
\chi_{S S, v a c}^{(0)}\left(-q_{E}^{2}\right)=I_{1, v a c}\left(M^{2}\right)+\left(q_{E}^{2}+4 M^{2}\right) I_{2, v a c}\left(M^{2}, q_{E}^{2}\right) .
$$

Here $q_{E}^{2}=q^{2}+q_{4}^{2}=q_{1}^{2}+\ldots+q_{4}^{2}$ and the functions $I_{1, v a c}\left(M^{2}\right)$ and $I_{2, v a c}\left(M^{2}, q_{E}^{2}\right)$ are defined by the expressions below

$$
\begin{gathered}
I_{1, v a c}\left(M^{2}\right)=8 N_{c} \int \frac{d^{4} p_{E}}{(2 \pi)^{4}} \frac{1}{p_{E}^{2}+M^{2}}, \\
I_{2, v a c}\left(M^{2}, q_{E}^{2}\right)=-4 N_{c} \int \frac{d^{4} p_{E}}{(2 \pi)^{4}} \frac{1}{\left[\left(p_{E}+q_{E} / 2\right)^{2}+M^{2}\right]\left[\left(p_{E}-q_{E} / 2\right)^{2}+M^{2}\right]},
\end{gathered}
$$

where $d^{4} p_{E}=d^{3} p d p_{4}$.

Since the integrals (17) and (18) diverge, the quantities (14), (15) and (16) are not well defined. In order to obtain finite results, we apply a modified Pauli-Villars subtraction scheme, where the functions $I_{1, v a c}\left(M^{2}\right)$ and $I_{2, v a c}\left(M^{2}, q_{E}^{2}\right)$ are replaced by

$$
I_{1, v a c}\left(M^{2}\right) \rightarrow I_{1, v a c}^{R}\left(M^{2}\right)=\sum_{i=0}^{N} A_{i} I_{1, v a c}\left(\Lambda_{i}^{2}\right)
$$

and

$$
I_{2, v a c}\left(M^{2}, q_{E}^{2}\right) \rightarrow I_{2, v a c}^{R}\left(M^{2}, q_{E}^{2}\right)=\sum_{i=0}^{N} A_{i} I_{2, v a c}\left(\Lambda_{i}^{2}, q_{E}^{2}\right)
$$


In Eqs. (19) and (20) $N$ is the number of subtractions, $A_{0}=1$ and $\Lambda_{0}=M$. The coefficients $A_{i}$, for $i>0$, have to be chosen in such a way as to provide the finite result for $I_{1, v a c}^{R}$ and $I_{2, v a c}^{R}$. Moreover, the correlation functions should be well behaved at infinity (for $q \rightarrow \infty)$, to guarantee the existence of the Fourier transform (10). These requirements lead to the following set of constraints

$$
\sum_{i=0}^{N} A_{i}=0, \quad \sum_{i=0}^{N} A_{i} \Lambda_{i}^{2}=0, \quad \ldots \quad, \sum_{i=0}^{N} A_{i} \Lambda_{i}^{2(N-1)}=0 .
$$

Following [7, 11] one finds

$$
I_{1, v a c}^{R}\left(M^{2}\right)=\frac{N_{c}}{2 \pi^{2}} \sum_{i=0}^{N} A_{i} \Lambda_{i}^{2} \ln \Lambda_{i}^{2}
$$

and

$$
I_{2, v a c}^{R}\left(M^{2}, q_{E}^{2}\right)=\frac{N_{c}}{2 \pi^{2}} \sum_{i=0}^{N} A_{i}\left[\frac{2 \Lambda_{i}}{q_{E}} \sqrt{1+\left(\frac{q_{E}}{2 \Lambda_{i}}\right)^{2}} \ln \left(\sqrt{1+\left(\frac{q_{E}}{2 \Lambda_{i}}\right)^{2}}+\frac{q_{E}}{2 \Lambda_{i}}\right)+\ln \Lambda_{i}\right]
$$

Substituting Eqs. (22) and (23) into (14), (15) and (16) yields finite expressions for the vacuum parts of the condensate and the zeroth-order correlation functions. The correlation functions evaluated in such a way are functions of the squared Euclidean momentum. This form is convenient for doing the Fourier transform (10). In this case we set $q_{4}=0$ and we use Eq. (23) with the substitution $q_{E}^{2}=q^{2}$.

In order to compute the dynamical masses, we need the function $I_{2, v a c}^{R}\left(M^{2},-\omega^{2}\right)$. This can be obtained by performing the analytic continuation of the function defined on the right hand side of Eq. (23). The substitution $q_{E} \rightarrow i \omega \pm \epsilon$ (for $\omega>0$ ) leads to the following result [11]

$$
\begin{aligned}
& I_{2, v a c}^{R}\left(M^{2},-\omega^{2} \pm i \epsilon\right)=\frac{N_{c}}{2 \pi^{2}} \sum_{i=0}^{N} A_{i}\left\{\Theta ( 2 \Lambda _ { i } - \omega ) \left[\frac{2 \Lambda_{i}}{\omega} \sqrt{1-\left(\frac{\omega}{2 \Lambda_{i}}\right)^{2}} \arcsin \left(\frac{\omega}{2 \Lambda_{i}}\right)\right.\right. \\
& \left.\left.+\ln \Lambda_{i}\right]+\Theta\left(\omega-2 \Lambda_{i}\right)\left[\frac{2 \Lambda_{i}}{\omega} \sqrt{\left(\frac{\omega}{2 \Lambda_{i}}\right)^{2}-1}\left(\operatorname{arcosh}\left(\frac{\omega}{2 \Lambda_{i}}\right) \pm \frac{i \pi}{2}\right)+\ln \Lambda_{i}\right]\right\} .
\end{aligned}
$$

iii) Matter parts

Let us now consider the matter parts of the physical quantities of interest. In the case of the condensate we find, using Eqs. (4) and (11),

$$
\langle\bar{q} q\rangle_{m a t}=-\frac{M}{2} I_{1, m a t}\left(M^{2}\right)
$$


where

$$
I_{1, \text { mat }}\left(M^{2}\right)=8 i N_{c} \oint_{\mathcal{C}} \frac{d p^{0}}{2 \pi} \int \frac{d^{3} p}{(2 \pi)^{3}} \frac{1}{p_{0}^{2}-\mathbf{p}^{2}-M^{2}} .
$$

To evaluate the function $I_{1, \text { mat }}\left(M^{2}\right)$ we collect the contributions from the poles within the contour $\mathcal{C}$ (see Fig. 1)

$$
I_{1, \text { mat }}\left(M^{2}\right)=-\frac{2 N_{c}}{\pi^{2}} \int_{0}^{\infty} \frac{d p p^{2}}{\omega_{p}} \theta\left(\mu-\omega_{p}\right)=-\frac{2 N_{c}}{\pi^{2}} \int_{0}^{p_{F}} \frac{d p p^{2}}{\omega_{p}} .
$$

Here $\theta(x)$ is the step function, $\omega_{p}=\sqrt{p^{2}+M^{2}}$, and $p_{F}$ is the Fermi momentum of the quarks, i.e., $p_{F}=\sqrt{\mu^{2}-M^{2}}$.

As was pointed out in Section 2, the matter parts of the zeroth-order correlation functions depend separately on $\omega^{2}$ and $q^{2}$. Nevertheless, for the extreme cases of a purely timelike $\left(Q^{\mu}=(\omega, 0)\right)$ or a purely spacelike momentum $\left(Q^{\mu}=(0, \mathbf{q})\right)$ the following decompositions, analogous to those obtained in vacuum, are possible

$$
\chi_{P P, \text { mat }}^{(0)}\left(\omega^{2}, q^{2}\right)=I_{1, \text { mat }}\left(M^{2}\right)-Q^{2} I_{2, \text { mat }}\left(M^{2}, \omega^{2}, q^{2}\right)
$$

and

$$
\chi_{S S, \text { mat }}^{(0)}\left(\omega^{2}, q^{2}\right)=I_{1, \text { mat }}\left(M^{2}\right)-\left(Q^{2}-4 M^{2}\right) I_{2, \text { mat }}\left(M^{2}, \omega^{2}, q^{2}\right) .
$$

Here the function $I_{2, \text { mat }}\left(M^{2}, \omega^{2}, q^{2}\right)$ is given by the integral

$$
I_{2, \text { mat }}\left(M^{2}, \omega^{2}, q^{2}\right)=4 i N_{c} \oint_{\mathcal{C}} \frac{d p^{0}}{2 \pi} \int \frac{d^{3} p}{(2 \pi)^{3}} \frac{1}{\left[(p+Q / 2)^{2}-M^{2}\right]\left[(p-Q / 2)^{2}-M^{2}\right]}
$$

The calculation of $I_{2, \text { mat }}\left(M^{2}, \omega^{2}, q^{2}\right)$ proceeds in the same way as the calculation of the function $I_{1, \text { mat }}\left(M^{2}\right)$. Now, as remarked above, the integral over $p^{0}$ is evaluated for imaginary $\omega$ and subsequently analytically continued to real frequencies. The final results (for $\omega>0$ ) are

$$
\begin{aligned}
I_{2, \text { mat }}\left(M^{2}, \omega^{2} \pm i \epsilon, 0\right) & =\frac{N_{c}}{2 \pi^{2}} \int_{0}^{p_{F}} \frac{d p p^{2}}{\omega_{p}} \frac{1}{\omega_{p}^{2}-(\omega \pm i \epsilon)^{2} / 4} \\
& =\frac{N_{c}}{2 \pi^{2}}\left[\log \left(\frac{p_{F}+\omega_{F}}{M}\right)-\mathcal{F}\left(M^{2}, \omega^{2} \pm i \epsilon\right)\right]
\end{aligned}
$$

and

$$
\begin{aligned}
I_{2, \text { mat }}\left(M^{2}, 0, q^{2}\right) & =-\frac{N_{c}}{2 q \pi^{2}} \int_{0}^{p_{F}} \frac{d p p}{\omega_{p}} \ln \left|\frac{2 p-q}{2 p+q}\right|= \\
& =-\frac{N_{c}}{2 q \pi^{2}} \mathcal{G}\left(M^{2}, q^{2}\right)
\end{aligned}
$$


where $\omega_{F}=\sqrt{p_{F}^{2}+M^{2}}$,

$$
\begin{array}{rlrl}
\mathcal{F}\left(M^{2}, \omega^{2} \pm i \epsilon\right) & =-\frac{1}{\omega}\left[\sqrt{4 M^{2}-\omega^{2}} \arctan \left(\frac{p_{F} \omega}{\omega_{F} \sqrt{4 M^{2}-\omega^{2}}}\right)\right] & (\omega<2 M) \\
& =-\frac{1}{\omega} \sqrt{\omega^{2}-4 M^{2}} \ln \left[\frac{\omega_{F} \sqrt{\omega^{2}-4 M^{2}}+p_{F} \omega}{\omega_{F} \sqrt{\omega^{2}-4 M^{2}}-p_{F} \omega}\right] \quad(\omega>2 M) \\
& \pm i \frac{\pi}{2 \omega} \theta(\omega-2 M) \theta\left(2 \omega_{F}-\omega\right) \sqrt{\omega^{2}-4 M^{2}} &
\end{array}
$$

and

$$
\begin{aligned}
\mathcal{G}\left(M^{2}, q^{2}\right) & =\left(\frac{\sqrt{q^{2}+4 M^{2}}}{2}-\omega_{F}\right) \ln \left|\frac{2 p_{F}+q}{2 p_{F}-q}\right| \\
& +\frac{\sqrt{q^{2}+4 M^{2}}}{2} \ln \left[\frac{2 M^{2}+q p_{F}+\omega_{F} \sqrt{q^{2}+4 M^{2}}}{2 M^{2}-q p_{F}+\omega_{F} \sqrt{q^{2}+4 M^{2}}}\right] \\
& -q \ln \left[\frac{p_{F}+\omega_{F}}{M}\right] .
\end{aligned}
$$

We need the analytic structure of the function $I_{2, \text { mat }}\left(M^{2}, 0, q^{2}\right)$ in the whole complex $q=|\mathbf{q}|$ plane. To this end we represent $I_{2, \text { mat }}\left(M^{2}, 0, q^{2}\right)$ as a sum of two functions, namely

$$
I_{2, \text { mat }}\left(M^{2}, 0, q^{2}\right)=I_{2, \text { mat }}^{(+)}(q)+I_{2, \text { mat }}^{(-)}(q)
$$

where

$$
I_{2, \text { mat }}^{( \pm)}(z)=-\frac{N_{c}}{4 z \pi^{2}} \int_{0}^{p_{F}} \frac{d p p}{\omega_{p}} \ln \frac{2 p-z \pm i \epsilon}{-2 p-z \pm i \epsilon} .
$$

At the end of the calculations we let the infinitesimal $\epsilon$ go to zero. The functions $I_{2, m a t}^{( \pm)}(z)$ have logarithmic cuts parallel to the real axis and stretching from $-2 p_{F} \pm i \epsilon$ to $+2 p_{F} \pm i \epsilon$. At the respective cut the imaginary part of the function $I_{2, m a t}^{( \pm)}(z)$ is discontinuous. On the physical Riemann sheet, this amounts to a change in sign:

$$
\operatorname{Im} I_{2, m a t}^{(+)}\left(q_{R}+i \delta_{ \pm}\right)=\mp \frac{N_{c}}{4 q_{R} \pi}\left[\sqrt{p_{F}^{2}+M^{2}}-\sqrt{\frac{q_{R}^{2}}{4}+M^{2}}\right],
$$

where $q_{R}$ is real and $-2 p_{F}<q_{R}<2 p_{F}, \delta_{+}=2 \epsilon$ and $\delta_{-}=0$. Thus, for the upper sign one is above the cut of $I_{2, \text { mat }}^{(+)}$and for the lower sign below. Obviously the imaginary part of $I_{2, \text { mat }}^{(-)}$above and below its cut is equal to that of $I_{2, \text { mat }}^{(+)}$above and below its cut. This can be summarized by the following equation

$$
\begin{aligned}
& \operatorname{Im} I_{2, \text { mat }}^{(-)}\left(z=q_{R}-i \delta_{\mp}\right)=\operatorname{Im} I_{2, \text { mat }}^{(+)}\left(z=q_{R}+i \delta_{ \pm}\right) \\
& \operatorname{Im} I_{2, \text { mat }}^{(-)}\left(z=q_{R}-i \delta_{ \pm}\right)=-\operatorname{Im} I_{2, \text { mat }}^{(+)}\left(z=q_{R}+i \delta_{ \pm}\right)
\end{aligned}
$$

Consequently, the cuts are arranged in such a way that for $z$ on the real axis, the imaginary part of $I_{2, \text { mat }}^{(+)}(z)$ cancels that of $I_{2, \text { mat }}^{(-)}(z)$ and $I_{2, \text { mat }}\left(M^{2}, 0, q^{2}\right)$ is real for real $q$ as it should be. 
Using Eqs. (32),(34),(35) and (36) we can find the analytic continuation of $I_{2, \text { mat }}\left(M^{2}, 0, q^{2}\right)$ to purely imaginary values of $q$. Substituting $q=i k$ we find

$$
I_{2, \text { mat }}\left(M^{2}, 0,-k^{2}\right)=-\frac{N_{c}}{k \pi^{2}} \int_{0}^{p_{F}} \frac{d p p}{\omega_{p}} \arctan \left(\frac{2 p}{k}\right) .
$$

The analytic form of $I_{2, \text { mat }}\left(M^{2}, 0,-k^{2}\right)$ is easily obtained from (32) and (34) by analytic continuation.

The integrals (26) and (30), which define the functions $I_{1, \text { mat }}$ and $I_{2, \text { mat }}$ are finite. Consequently, we are not forced to regularize them. However, if we consider the cutoff to be an intrinsic property of the quark-quark interaction, there is no reason not to regularize the matter parts in the same way as the vacuum parts. In such an approach, all fermion loops vanish when $\mu \rightarrow \infty$ (or $T \rightarrow \infty$ ) due to a cancellation between the vacuum and matter parts, as was discussed in the case of finite temperatures [11]. This is a desirable property, since it guarantees that the condensate and the zeroth-order correlation functions are well behaved in the high density/temperature limit. Consequently, we replace the matter parts by their regularized counterparts

$$
I_{1, \text { mat }}\left(M^{2}\right) \rightarrow I_{1, \text { mat }}^{R}\left(M^{2}\right)=\sum_{i=0}^{N} A_{i} I_{1, \text { mat }}\left(\Lambda_{i}^{2}\right)
$$

and

$$
I_{2, m a t}\left(M^{2}, \omega^{2}, q^{2}\right) \rightarrow I_{2, m a t}^{R}\left(M^{2}, \omega^{2}, q^{2}\right)=\sum_{i=0}^{N} A_{i} I_{2, m a t}\left(\Lambda_{i}^{2}, \omega^{2}, q^{2}\right) .
$$

We note that for $\mu<2 \Lambda_{1}$, which usually includes the region of interest to us, the additional terms appearing for $i>0$ in Eqs. (40) and (41) vanish. Therefore, the regularization of the matter parts become effective only at very large densities.

The structure of Eqs. (14) - (16), (25), (28) and (29) suggests that the following definitions will be useful

$$
I_{1}\left(M^{2}\right)=I_{1, v a c}^{R}\left(M^{2}\right)+I_{1, \text { mat }}^{R}\left(M^{2}\right)
$$

and

$$
I_{2}\left(M^{2}, \omega^{2}, q^{2}\right)=I_{2, v a c}^{R}\left(M^{2}, q^{2}-\omega^{2}\right)+I_{2, \text { mat }}^{R}\left(M^{2}, \omega^{2}, q^{2}\right) .
$$

Eqs. (31) - (38), (39) have a very similar form to the expressions found in [11. The formal correspondence can be obtained by the replacement of the functions $\left[\exp \left(\beta \omega_{p}\right)+1\right]^{-1}$ by $\frac{1}{2} \theta\left(\mu-\omega_{p}\right)$. Nevertheless, there is a crucial difference between the present calculation and [11]: here the cut along the real axis is finite, which leads to a completely different behaviour of the correlation functions at large distances.

\section{Density dependence of the quark and meson masses.}

Using Eqs. (5), (12), (14), (25) and (42) we find that

$$
M=m+M G_{S} I_{1}\left(M^{2}\right)
$$


Eq. (44) is the gap equation determining the constituent quark mass $M$. This equation determines $M$ as a function of the chemical potential $\mu$. The dynamic masses of pion and sigma are obtained from Eq. (8). Assuming that the gap equation has a non-trivial solution, $M \neq 0$, we obtain

$$
\frac{m}{M}+m_{d y n, \pi}^{2} I_{2}\left(M^{2}, m_{d y n, \pi}^{2}, 0\right)=0
$$

and

$$
\frac{m}{M}+\left(m_{d y n, \sigma}^{2}-4 M^{2}\right) I_{2}\left(M^{2}, m_{d y n, \sigma}^{2}, 0\right)=0
$$

If the current quark mass is zero, the pion is massless, i.e., a Goldstone boson and the mass of sigma is simply $2 M$.

We note that Eqs. (45) and (46) are correct not only in vacuum but also at finite density, as long as a non-trivial solution to the gap equation exists. Nevertheless, as follows from Eqs. (24) and (31), the correlation functions have cuts for arguments larger than $2 M$. At high densities the meson poles merge with the quark-antiquark cuts, which means that in this model the mesons can decay into $q \bar{q}$ pairs. Thus, at these densities there are no isolated poles which can be identified with the mass of the pion or the sigma. We circumvent this difficulty by defining the mass as the zero of the real part of Eq. (8), i.e.,

$$
1-G_{S} \operatorname{Re} \chi_{A A}^{(0)}\left(m_{d y n}^{2}, 0\right)=0 .
$$

We note that by using Eq. (47) we implicitly neglect the $q \bar{q}$ widths of the mesons. This seems reasonable, since these widths are non-zero only because the Nambu-Jona-Lasinio model lacks confinement.

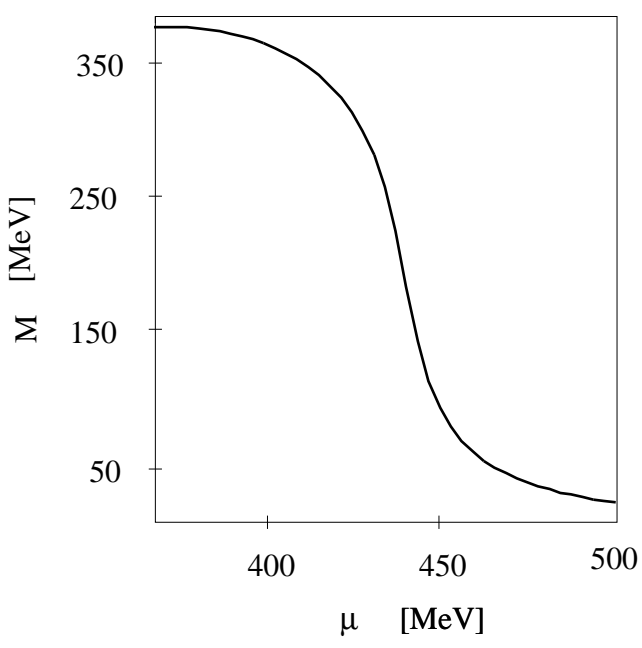

Figure 2: Constituent quark mass $M$ as a function of the quark chemical potential $\mu$. 
In Figs. 2 and 3 we show the density dependence of the constituent quark mass and of the dynamic masses of mesons. We use the same values of the parameters as in 11$]$. Consequently, our results for $\mu=0$ coincide with the $T=0$ results of [11]. The values of the three regulating masses are: $\Lambda_{1}=680 \mathrm{MeV}, \Lambda_{2}=2.1 \Lambda_{1}$ and $\Lambda_{3}=2.1 \Lambda_{2}$. The coupling constant $G_{S}=0.75 \mathrm{fm}^{2}$ and the current quark mass $m=8.56 \mathrm{MeV}$. In vacuum we find the constituent quark mass $M=M_{0}=376 \mathrm{MeV}$, the pion mass $m_{d y n, \pi}=138 \mathrm{MeV}$, and the sigma mass $m_{d y n, \sigma}=760 \mathrm{MeV}$. The corresponding value of the quark condensate is $\langle\bar{q} q\rangle^{1 / 3}=$ $-214 \mathrm{MeV}$ and of the pion decay constant $f_{\pi}=94 \mathrm{MeV}$. The latter can be obtained from the Gell-Mann - Oakes - Renner relation: $f_{\pi}^{2} m_{\pi}^{2}=-2 m\langle\bar{q} q\rangle$.

We note that at $T=0$ all values of the chemical potential in the range $0<\mu<M_{0}$ are equivalent and correspond to the physical vacuum. The reason is that the chemical potential $\mu$ is the energy needed to add a quark to the system. Clearly $\mu$ must exceed the minimal energy, the vacuum rest mass of a quark $M_{0}$, before the Fermi sea of quarks begins to be populated.

In Fig. 2, one can see that the constituent quark mass decreases with the increasing value of the chemical potential. At the same time, see Fig. 3, the dynamic masses of pion and sigma become degenerate. This fact indicates restoration of the chiral symmetry at high density. The overall $\mu$-dependence shown in Figs. 2 and 3 is analogous to the $T$-dependence studied in [11].

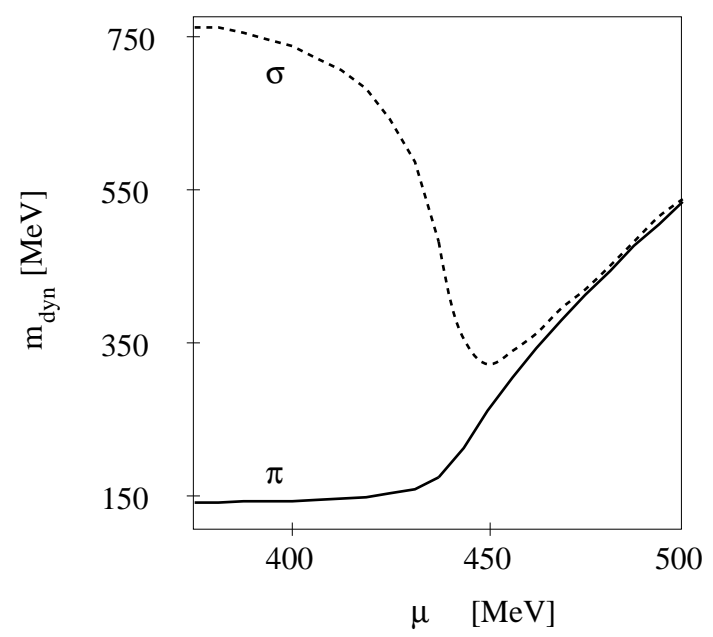

Figure 3: Pion (solid line) and sigma (dashed line) dynamic masses plotted as functions of the quark chemical potential $\mu$.

The change of the in-medium constituent quark mass $M$ implies, through Eq. (5), a modification of the value of the quark condensate. The calculation of the decrease of the condensate with increasing baryon density in the framework of the NJL model is instructive, since there are model independent estimates of this quantity, which impose constraints on the in-medium behaviour of the condensate. Using the Hellmann-Feynman theorem and the 
Gell-Mann - Oakes - Renner relation, one finds [22, 23] to leading order in the density

$$
\frac{\langle\bar{q} q\rangle}{\langle\bar{q} q\rangle_{0}}=1-\frac{\Sigma_{\pi N}}{m_{\pi}^{2} f_{\pi}^{2}} \rho,
$$

where $\Sigma_{\pi N}$ is the pion nucleon sigma commutator, $\langle\bar{q} q\rangle_{0}$ is the vacuum value of the quark condensate, and $\rho$ is the baryon density. The baryon density is for two flavours given by $\rho=2 p_{F}^{3} / 3 \pi^{2}$, where $p_{F}=\sqrt{\mu^{2}-M^{2}}$ is the quark Fermi momentum. We compute the pion nucleon sigma term by using the relation [23]

$$
\frac{1}{3} \Sigma_{\pi N}=\Sigma_{\pi q}=m \frac{d M_{0}}{m}
$$

where we assume that the $\pi N$ sigma term is simply the sum of the $\pi q$ sigma terms, like in the naive quark model. For the parameters obtained above we find $\Sigma_{\pi N}=18 \mathrm{MeV}$, which, using Eq. (48), implies that the condensate is reduced by $15 \%$ at the saturation density of nuclear matter, $\rho_{0}=0.17 \mathrm{fm}^{-3}$. In Fig. 4 we show the numerical results for the quark condensate (normalized to its vacuum value) as a function of the baryon density (normalized to the saturation density). We note that the calculation agrees with the low-density theorem (48), when we use the sigma commutator obtained within the model. Since the sigma term is much smaller than the empirical value of $45 \mathrm{MeV}$, the density dependence of the quark condensate is too weak. Nevertheless, the fact that Eq. (48) is satisfied, shows that the calculation is consistent.

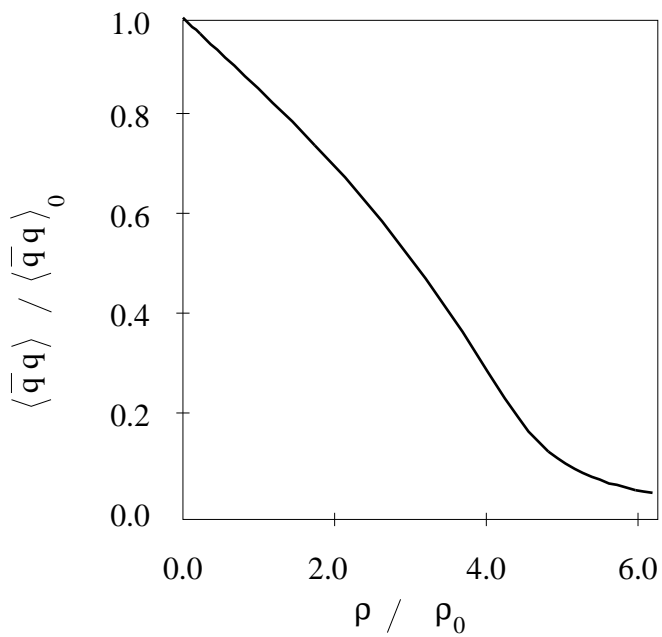

Figure 4: Ratio of the in-medium quark condensate to the vacuum one, plotted as a function of baryon density. 


\section{Static correlation functions in space}

i) Analytic structure in complex momentum space

In this Section we discuss our results on the spatial dependence of static correlation functions. By deforming the integration contour, the Fourier transform (10) can be rewritten as a sum of a few contributions, which are due to the singularities of the correlation function in the complex $q$-plane. The results of Section 3 indicate that $\chi_{v a c}^{(0)}\left(-q^{2}\right)$ has cuts (in the following we refer to these as the vacuum cuts) along the imaginary axis starting at $q= \pm 2 i M$ and going to $\pm i \infty$. On the other hand the function $\chi_{\text {mat }}^{(0)}\left(0, q^{2}\right)$ has cuts (matter cuts) which are parallel to the real axis. Thus, the full correlation $\chi_{A A}\left(-q^{2}\right)$ function defined by Eq. (7) has at least these two cuts. Moreover, it can have poles for imaginary arguments in between the cuts. The general structure of these singularities is shown in Fig. 5.

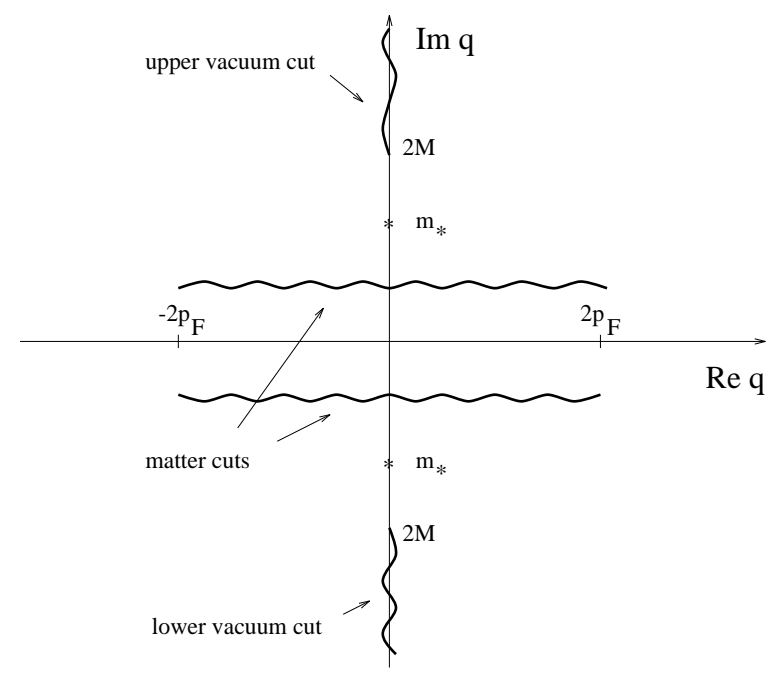

Figure 5: Structure of the singularities of the correlation functions in the complex $q=|\mathbf{q}|$ space.

The position of the pole is determined by

$$
1-G_{S} \chi_{A A}^{(0)}\left(0,-m_{*}^{2}\right)=0 .
$$

A pole can appear only in the interval $0<m_{*}<2 M$, since the vacuum cut starts at $m_{*}=2 M$. In vacuum, due to the Lorentz invariance, we find that $m_{*}=m_{d y n}$. On the other hand, for $\mu>M_{0}, m_{*} \neq m_{d y n}$ in general, because the functions $I_{2, m a t}\left(M^{2}, k^{2}, 0\right)$ and $I_{2, \text { mat }}\left(M^{2}, 0,-k^{2}\right)$ are different; compare Eqs. (31) and (39). The contribution of the pole to the Fourier transform is of course of the form $\sim \exp \left(-m_{*} r\right)$. However, there are additional contributions from the vacuum as well as the matter cuts. The integral around the vacuum cut can be written as follows 


$$
\chi_{A A}^{V C}(r)=-\frac{1}{4 \pi^{2} i r} \int_{2 M}^{\infty} d k k\left[\chi_{A A}\left(0,-k^{2}+i \epsilon\right)-\chi_{A A}\left(0,-k^{2}-i \epsilon\right)\right] e^{-k r}
$$

For large values of $r$, the exponential factor in (51) cuts down the integrand very quickly as a function of $k$. Thus, the remaining factors, which are slowly varying, can be approximated by their value at $k \approx 2 M$. Consequently, for $r \rightarrow \infty$ the contribution of the vacuum cut is also of the exponential form, $\sim \exp (-2 M r)$. The contribution of the matter cut is

$$
\chi_{A A}^{M C}(r)=\frac{1}{4 \pi^{2} i r} \int_{-2 p_{F}}^{+2 p_{F}} d q q\left[\chi_{A A}\left(0,\left[q+i \delta_{-}\right]^{2}\right)-\chi_{A A}\left(0,\left[q+i \delta_{+}\right]^{2}\right)\right] e^{i q r}
$$

where $\delta_{ \pm}$is defined below Eq. (37). The imaginary shift of the argument, $i \delta_{ \pm}$, denotes that the function should be calculated just above/below the matter cut. We note that in contrast to the finite temperature calculation [11], the contribution by the matter cut is in the present case represented by an integral over the finite range in $q$. This leads to a qualitatively different behaviour of the Fourier transform.

\section{ii) Results}

Let us start the presentation of the results by discussing the zero density case $(0<\mu<$ $\left.M_{0}\right)$. This is equivalent to the zero temperature case $(T=0)$ studied in [11]. In vacuum the matter cut vanishes and we are left with only one or two singularities which contribute to the Fourier transform. This allows for the simple analysis of the asymptotic behaviour of the correlation functions.

In the case of the pseudoscalar channel we find an isolated pole and a cut. Because of the Lorentz invariance, the pole coincides with the dynamical mass. For large values of $r$ the contribution from the pole, $\sim \exp \left(-m_{d y n, \pi} r\right)$, dominates over that of the vacuum cut, $\sim \exp \left(-2 M_{0} r\right)$. Consequently, the static correlation function in the pseudoscalar channel exhibits a pure exponential behaviour, whose decay rate is characterized by the pion screening mass: $m_{s c r, \pi}=m_{d y n, \pi}=138 \mathrm{MeV}$.

In the case of the scalar channel we do not have an isolated pole and the only contribution to the Fourier transform is due to the vacuum cut. The latter also leads to the exponential behaviour. In consequence the sigma screening mass equals $2 M_{0}$ and is a little bit smaller than the dynamic one computed using Eq. (47): $m_{s c r, \sigma}=752 \mathrm{MeV}$ and $m_{d y n, \sigma}=760 \mathrm{MeV}$.

Our numerical calculations, done in the interval $0.5 \mathrm{fm}<r<6 \mathrm{fm}$, confirm the results of the analysis carried out above: the correlation functions in both pseudoscalar and scalar channel decays exponentially with distance, although the numerically evaluated sigma screening mass turns out to be slightly larger than $2 M_{0}$. The discrepancy between the numerical and analytical results is due to the fact that numerically we are not able to compute at truly asymptotic distances.

Let us now discuss the finite density calculations. In the case of the pseudoscalar channel, we did the numerical calculations in the energy range $M_{0}=376 \mathrm{MeV} \leq \mu \leq 415 \mathrm{MeV}$. In Fig. 6 we show the corresponding correlation for $\mu=376,400$ and $410 \mathrm{MeV}$. In contrast to the exponential decay found in vacuum (solid line), the correlation function at finite density 
(dashed and dotted lines) oscillates, with a power-law decay of the amplitude. Its period decreases with the increasing value of the chemical potential. From the physical point of view, such oscillations are caused by the existence of a sharp Fermi surface.

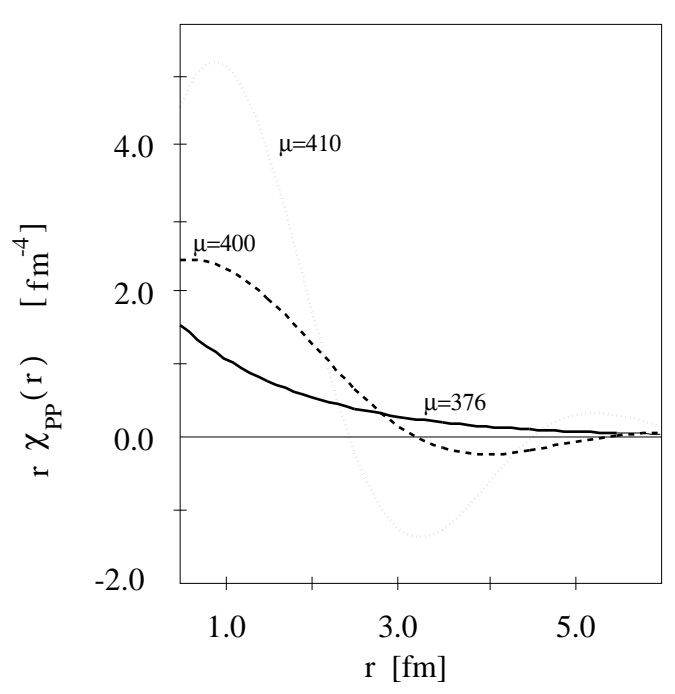

Figure 6: Correlation function in the pseudoscalar channel plotted for three different values of the chemical potential: $\mu=376 \mathrm{MeV}$ (solid line), $\mu=400 \mathrm{MeV}$ (dashed line), and $\mu=410 \mathrm{MeV}$ (dotted line).

The oscillatory behaviour of the correlation functions at finite density is well known for the non-relativistic degenerate electron gas, where the phenomenon is called Friedel oscillations [20]. These long range oscillations lead to many interesting phenomena like, e.g, broadening of the nuclear magnetic resonance lines. A characteristic feature of the Friedel oscillations (at very large distances) is their period $\delta r=\pi / p_{F}$. In our case the situation is similar; for very large values of $r$, the contribution from the matter cut dominates over the other ones. Since the cut extends over a finite range in $q\left(|q|<2 p_{F}\right)$ the correlation function oscillates with the period $\pi / p_{F}$ (at $\left.r \rightarrow \infty\right)$. Nevertheless, the numerical study of the correlation functions at very large distances is difficult because the amplitudes decrease with $r$. Consequently, we restrict the numerical calculations to the interval $0.5 \mathrm{fm}<r<6 \mathrm{fm}$.

So far we have discussed the results for $\mu<415 \mathrm{MeV}$. For larger values of the chemical potential (corresponding roughly to $\rho>\frac{1}{2} \rho_{0}$ ) the pseudoscalar correlation function acquires additional singularity on the real axis for $q \approx 2 p_{F}$. In the scalar channel, a similar singularity appears at even smaller densities $\left(\rho>\frac{1}{3} \rho_{0}\right)$. These singularities indicate instabilities of the ground state, which lead the system to states of lower energy. However, most likely the singularities are artifacts due to regularization procedure and do not correspond to physical instabilities. In order to check this point we have redone the calculation using a different regularization scheme with a three-dimensional cutoff. Using the parameters obtained in Ref. [18], we find no singularities on the real axis. This shows that these singularities are unphysical, since their presence depends on details in the formulation of the model. Thus, our regularization scheme can be used only at low densities. 
On the other hand, the three-dimensional cutoff regularization is unsatisfactory because it explicitly breaks Lorentz invariance. This obscures the relation between the correlation functions in timelike and spacelike regions. Moreover, in the three-dimensional cutoff scheme, one does not recover the well known screening of the correlation function at finite temperatures. Instead, the correlation function oscillates, much like the Friedel oscillations at $T=0$, with a period $\pi / \Lambda$, where $\Lambda$ is the momentum cutoff. We stress that this behaviour is an artifact, due to the finite range of momenta $(|q|<\Lambda)$ available in the Fourier transform.

Consequently, we stick to the covariant Pauli-Villars method, in spite of its shortcomings. However, since the results are inconclusive at high densities, $\rho \sim \rho_{0}$ and higher, we restrict the calculations to small densities $\rho<\frac{1}{3} \rho_{0}$. The physics at very large densities will be addressed in the following section using a different (although closely related) approach.

\section{Perturbative QCD calculation}

The formalism developed so far can also be used to study correlation functions in perturbative QCD. In this case the leading term in the meson correlation function is the lowest order quark loop contribution. Thus, at large densities the correlation function in r-space is given by

$$
\chi_{A A}^{(0)}(r)=\frac{1}{4 \pi^{2} i r} \int_{-\infty}^{\infty} d q q \chi_{A A}^{(0)}\left(0, q^{2}\right) e^{i q r}
$$

The Fourier transforms of the vacuum $\chi_{A A, v a c}^{(0)}\left(0, q^{2}\right)$ and matter $\chi_{A A, m a t}^{(0)}\left(0, q^{2}\right)$ parts define the functions $\chi_{A A, v a c}^{(0)}(r)$ and $\chi_{A A, m a t}^{(0)}(r)$, respectively, with

$$
\chi_{A A}^{(0)}(r)=\chi_{A A, v a c}^{(0)}(r)+\chi_{A A, m a t}^{(0)}(r) .
$$

The Fourier transform (53) can be computed analytically. Using the methods developed in [0], we find that

$$
\chi_{P P, v a c}^{(0)}(r)=\frac{N_{c}}{2 \pi^{3} r^{3}} \sum_{i=0}^{N} A_{i} \Lambda_{i}^{2}\left[3 K_{2}\left(2 \Lambda_{i} r\right)+2 \Lambda_{i} r K_{1}\left(2 \Lambda_{i} r\right)\right] .
$$

where $K_{1}$ and $K_{2}$ are the modified Bessel functions [24]. The matter piece, on the other hand, is given by

$$
\chi_{P P, \text { mat }}^{(0)}(r)=\frac{N_{c}}{4 \pi^{3} r}\left(\frac{2}{r^{3}}-\frac{2}{r^{2}} \frac{\partial}{\partial r}+\frac{1}{r} \frac{\partial^{2}}{\partial r^{2}}\right)\left[G_{1}(r)+G_{2}(r)\right]
$$

where

$$
G_{1}(r)=\frac{\partial}{\partial r} \sum_{i=0}^{N} \frac{A_{i}}{2} \int_{0}^{\infty} \frac{d p}{\sqrt{p^{2}+\Lambda_{i}^{2}}} \cos (2 p r)=-\sum_{i=0}^{N} A_{i} \Lambda_{i} K_{1}\left(2 \Lambda_{i} r\right)
$$

and

$$
G_{2}(r)=-\frac{\partial}{\partial r} \sum_{i=0}^{N} \frac{A_{i}}{2} \int_{p_{F}}^{\infty} \frac{d p}{\sqrt{p^{2}+\Lambda_{i}^{2}}} \cos (2 p r)
$$


The properties of the modified Bessel functions [25] imply that the vacuum part (55) is exactly canceled by the $G_{1}(r)$ term of the matter part (56). The cancellation is quite general and in fact independent of the number of subtractions $N$ and of the values of the regulating masses $\Lambda_{i}$.

The vacuum part of the correlation function $\chi_{A A, v a c}^{(0)}\left(0, q^{2}\right)$ is divergent and must be regularized. Nevertheless, the Fourier transform $\chi_{A A, v a c}^{(0)}(r)$ remains finite for $r>0$ also when one sends the cutoff masses to infinity. To see how this works we consider the Fourier integral of the regularized vacuum correlation function. This is well defined, so that the integration contour can be deformed into a path around the cut on the positive imaginary axis starting at $p=2 i M$, where $M$ is the quark mass. The integral around the cut converges for $r>0$ and the contributions of the regularization terms, which correspond to cuts starting at $p=2 i \Lambda_{i}$ are exponentially suppressed. Consequently, the role of the regularization terms is to make the Fourier integral finite and thus to make the deformation of the contour possible. After the contour has been deformed, the integral is finite, for $r>0$, even when one sends the cutoff masses to infinity. In the limit $\Lambda_{i} \rightarrow \infty$ and $M \rightarrow 0$, we find

$$
G_{2}(r)=\frac{\cos \left(2 p_{F} r\right)}{2 r}
$$

The form of $G_{2}(r)$ implies that the correlation function $\chi_{P P}^{(0)}(r)$ is not screened but oscillates in space with a period $\delta r=\pi / p_{F}$. For massless quarks, the behaviour of $\chi_{S S}^{(0)}(r)$ is identical, since the two channels are degenerate in this limit.

\section{Summary}

In this paper, we have studied the structure of static meson correlation functions at finite baryon density within the NJL model. We have restricted our work to the pseudoscalar and scalar channels.

In general the vacuum correlation functions in the meson channels are screened and, due to Lorentz invariance, the screening mass of a stable meson equals its dynamical mass. For unstable mesons the screening mass equals the mass of the branch point, since the asymptotic form of the correlation function picks out the lowest lying singularity. As demonstrated in our previous work [11], these results are qualitatively reproduced by the Nambu-Jona-Lasinio model: the pion screening mass is equal to its dynamic mass, whereas the sigma screening mass is $2 M_{0}$, where $M_{0}$ is the constituent quark mass in vacuum. [The latter result is of course an artifact of the NJL model, which lacks confinement and of the approximation, which lacks a coupling of the sigma to the two-pion continuum. In a more realistic treatment the screening mass in the sigma channel should equal $2 m_{\pi}$.] At finite temperature and zero density, the correlation functions are again exponentially damped, although the screening and dynamic masses differ. However, as we have demonstrated here, the correlation functions at $T=0$ and finite density differ qualitatively from those in vacuum: they exhibit long ranged oscillations, of the Friedel type, rather than exponential damping.

In order to understand this effect, we carefully explored the analytic structure of the correlation functions. We found that the appearance of the oscillations is connected with the existence of a cut of finite range in the complex momentum plane. This cut is responsible for the leading contribution to the correlation function at large distances. The length of the cut, 
which is proportional to the Fermi momentum of the constituent quarks, is reflected in the oscillation period at large distances $\delta r=\pi / p_{F}$. Consequently this form of the correlation function is quite general and is expected in all normal Fermi liquids. In particular, the existence of the oscillations is independent of whether the basic fermionic degrees of freedom are quarks or nucleons. Therefore, we feel the oscillatory behaviour of the correlation function at finite density will not change qualitatively by confinement.

The fact that the correlation function exhibits Friedel type oscillations indicates that at finite density it is impossible to obtain the information about timelike excitations by studying spacelike correlation functions. Their long distance behaviour is dominated by low lying particle-hole excitations, and consequently not connected with the dynamical mass.

We find, in agreement with the low density theorem [22, 23], that the quark condensate is reduced as the density is increased and eventually almost vanishes, implying that the chiral symmetry is restored at high densities. This, and the fact that the dynamical masses of chiral partners (here $\pi$ and $\sigma$ ) become degenerate with increasing density, seems to indicate that the NJL model offers a consistent description of the restoration of chiral symmetry at finite baryon density. Nevertheless, the spurious singularities we encountered in the correlation functions, suggest that the model in the present formulation cannot be naively used for densities $\rho>\frac{1}{3} \rho_{0}$. Therefore, we complemented our investigation with a calculation in perturbative QCD, which again indicates the presence of oscillations also at very high densities.

\section{Acknowledgment}

One of us (W. F.) would like to thank the Theory Group at GSI for very warm hospitality. This research was supported in part by the Polish State Committee for Scientific Research under Grant No. 2 P03B 18809 and by the Stiftung für Deutsch-Polnische Zusammenarbeit.

\section{References}

[1] E. Shuryak, Rev. Mod. Phys. 65 (1993) 1

[2] C. DeTar and J. Kogut, Phys. Rev. Lett. 59 (1987) 399; Phys. Rev. D36 (1987) 2828

[3] S. Gottlieb, W. Liu, R. L. Renken, R. L. Sugar and D. Touissant, Phys. Rev. Lett. 59 (1987) 1881, Nucl. Phys. A498 (1989) 435c

[4] A. Gocksch, P. Rossi and M. Heller, Phys. Lett. B205 (1988) 334

[5] K. Born, S. Gupta, A. Irbäck, F. Karsch, E. Laermann, B. Peterson and H. Satz, Phys. Rev. Lett. 67 (1991) 302

[6] V. L. Eletskii and B. L. Ioffe, Sov. J. Nucl. Phys. 48 (1988) 384

[7] W. Florkowski and B.L. Friman, Zeit f. Physik A347 (1994) 271

[8] A. Gocksch, Phys. Rev. Lett. 67 (1991) 1701

[9] T. H. Hanson and I. Zahed, Nucl. Phys. B374 (1992) 277

[10] V. Koch, E. V. Shuryak, G. E. Brown and A. D. Jackson, Phys. Rev. D46 (1992) 3169 
[11] W. Florkowski and B.L. Friman, Acta Phys. Pol. B25 (1994) 49

[12] Y. Nambu and G. Jona-Lasinio, Phys. Rev. 122 (1961) 345; 124 (1961) 246

[13] U. Vogl and W. Weise, Prog. Part. Nucl. Phys. 27 (1991) 195; S. P. Klevansky, Rev. Mod. Phys. 64 (1992) 649; M.K. Volkov, Phys. Part. Nucl. 24 (1993) 35; T. Hatsuda and T. Kunihiro, Phys. Rep. 247 (1994) 221

[14] T. Hatsuda and T. Kunihiro, Phys. Rev. Lett. 55 (1985) 158

[15] V. Bernard, U.-G. Meissner and I. Zahed, Phys. Rev. D36 (1987) 819

[16] T. Hatsuda and T. Kunihiro, Phys. Lett. B185 (1987) 304; Phys. Lett. B198 (1987) 126

[17] V. Bernard, U.-G. Meissner and I. Zahed, Phys. Rev. Lett. 59 (1987) 966

[18] J. Hüfner, S.P. Klevansky, P. Zhuang, and H. Voss, Ann. Phys. (N.Y.) 234 (1994) 225;

P. Zhuang, J. Hüfner, and S.P. Klevansky, Nucl. Phys. A576 (1994) 525

[19] G. 't Hooft, Phys. Rev. Lett. 37 (1976) 8; Phys. Rev. D14 (1976) 3432

[20] A. Fetter and J. Walecka, Quantum Theory of Many Particle Systems, (McGraw Hill, New York, 1971)

[21] J. Kapusta, Finite Temperature Field Theory, (Cambridge University Press, 1989)

[22] E.G. Drukarev and E.M. Levin, Nucl. Phys. A511 (1990) 679; Prog. Part. Nucl. Phys. 27 (1991) 77

[23] T.D. Cohen, R.J. Furnstahl, and D.K. Griegel, Phys. Rev. C45 (1992) 1881

[24] Handbook of Mathematical Functions (Dover Publications, New York, 1972), ed. M. Abramowitz and I. Stegun

[25] S. Gradshteyn and I. M. Ryzhik, Table of Integrals, Series, and Products, (Academic Press 1980) 